$07,14,18$

\title{
Описание степени усиления нанокомпозитов полимер/углеродные нанотрубки: предел „термита“
}

\author{
() Г.В. Козлов, И.В. Долбин \\ Кабардино-Балкарский государственный университет им. Х.М. Бербекова, \\ Нальчик, Россия \\ E-mail: i_dolbin@mail.ru \\ Поступила в Редакцию 10 марта 2021 г. \\ В окончательной редакции 5 апреля 2021 г. \\ Принята к публикации 24 апреля 2021 г.
}

Для описания свойств нанокомпозитов полиуретан/углеродные нанотрубки использована модель двухкомпонентных случайных смесей в пределе „термита“ (случайной сверхпроводящей сетки). Эта модель является корректной и дает достаточно точное количественное описание степени усиления рассматриваемых нанокомпозитов. Для получения точного описания рассматриваемой характеристики необходимо рассматривать структуру углеродных нанотрубок как кольцеобразные формирования, а критический индекс в пределе „термита“ определяется фрактальной размерностью этих формирований. Предложенная модель позволяет выяснить критерий получения высокомодульных нанокомпозитов этого класса.

Ключевые слова: нанокомпозит, углеродные нанотрубки, степень усиления, случайная смесь, предел „термита“, фрактальная размерность.

DOI: 10.21883/FTT.2021.09.51272.051

\section{1. Введение}

В наиболее простой и наглядной интерпретации полимерные нанокомпозиты можно представить как двухфазные случайные смеси, теоретические модели для описания которых рассмотрены в работе [1]. Существуют два предельных случая для описания таких систем:

1. случайная сетка резисторов (ССР) или предел „муравья“, который предполагает, что большая проводимость равна единице, а меньшая - равна нулю;

2. случайная сверхпроводящая сетка (ССC) или предел „термита“, который предполагает, что большая проводимость бесконечна, а меньшая - равна нулю.

В реальном случае для рассматриваемых в настоящей работе нанокомпозитов полиуретан/углеродные нанотрубки (ПУ/УНТ) предполагается реализация предела „Термита“ в силу большого различия модулей упругости (сопротивления прилагаемой нагрузке) компонент этого двухкомпонентного наноматериала: для матричного эластомерного полиуретана (ПУ) номинальный модуль упругости имеет величину порядка $10 \mathrm{MPa} \mathrm{[2],} \mathrm{тогда}$ как этот параметр для углеродных нанотрубок (УНТ) составляет величину порядка $1 \mathrm{TPa}$ [3], т. е. наблюдается различие сопротивления деформации компонент нанокомпозита на 5 порядков. В пределе ССС при приближении к порогу перколяции УНТ $p_{c}$ снизу проводимость $\Sigma$ стремится к бесконечности по закону [1]:

$$
\Sigma \sim\left(p_{c}-p\right)^{-s},
$$

где $p$ - концентрация проводящей фазы (в рассматриваемом случае - УНТ), $s$ - показатель.

Авторы [2] исследовали зависимость степени усиления (относительного модуля упругости) нанокомпозитов полиуретан/графен в рамках модели „термита“ (ССC) и получили хорошее соответствие с экспериментальными данными. Однако, хотя УНТ и графен являются сходными по своему строению нанонаполнителями, но они различаются размерностью исходных частиц: УНТ имеют линейную (одномерную) топологию, а графен плоскостную (двухмерную). В силу этого обстоятельства указанные сильно анизотропные нанонаполнители формируют в полимерной матрице нанокомпозитов разные типы своих агрегатов (кластеров) - УНТ образуют кольцеобразные формирования, структурно аналогичные макромолекулярным клубкам разветвленных полимерных цепей $[3,4]$, а графены - пачки коллинеарно расположенных пластин (тактоиды) $[2,4]$.

С учетом указанных различий как структуры исходных частиц нанонаполнителя, так и типа их агрегатов целью настоящей работы является описание зависимости степени усиления $E_{n} / E_{m}$ (где $E_{n}$ и $E_{m}-$ модули упругости нанокомпозита и матричного полимера, соответственно) от концентрации твердотельной (жесткой) компоненты, которой являются УНТ, в рамках теории перколяции с применением описанного выше предела „термита“ $[1]$.

\section{2. Результаты и обсуждение}

В настоящей работе выполнен теоретический анализ экспериментальных данных, полученных в работе [3], для нанокомпозитов ПУ/УНТ. В качестве матричного полимера использован эластомерный полиуретан марки Morthane PS455-203 производства фирмы Hunstman Polyurethane (США). В качестве нанонапол- 
нителя применялись многослойные углеродные нанотрубки (нановолокна) марки РRT-НT-19, производства Applied Science Inc. (США), имеющие наружный диаметр 50-120 nm и длину $10 \mathrm{mcm}$ и более [3].

Образцы нанокомпозитов ПУ/УНТ получены смешиванием растворов компонентов при содержании УНТ до 20 mass.\% в полярном растворителе (тетрахлорэтане) и последующим их приготовлением методом полива на стеклянную подложку. Затем образцы сушились в вакуумной печи при температуре $323 \mathrm{~K}$ до постоянной массы. Полученные образцы имели толщину $0.5-2.0 \mathrm{mcm}$. Механические испытания выполнены на приборе Tinius Olson H10KS при скорости ползуна $6 \mathrm{~mm} / \mathrm{min}$ и температуре $293 \mathrm{~K}$ [3].

Для применения перколяционной модели в виде предела „термита“ к рассматриваемому случаю нанокомпозитов ПУ/УНТ соотношение (1) следует переписать следующим образом:

$$
\frac{E_{n}}{E_{m}} \sim\left(\varphi_{c}-\varphi_{n}\right)^{-s}
$$

где $\varphi_{c}-$ порог перколяции УНТ, $\varphi_{n}-$ объемное содержание нанонаполнителя (жесткой фазы).

Рассмотрим методы оценки параметров, входящих в соотношение (2). Как хорошо известно [3], углеродные нанотрубки являются сильно анизотропным нанонаполнителем, чье аспектное отношение (отношение длина/диаметр) может достигать 100 и более. В этом случае наблюдается очень низкая (порядка 0.01) величина порога перколяции $\varphi_{c}[4,5]$. Однако, также хорошо известно [6], что в силу указанной выше высокой степени анизотропии и относительно низкого поперечного модуля упругости углеродные нанотрубки в полимерной матрице формируют кольцеобразные структуры радиуса $R_{\mathrm{CNT}}$ и поэтому их порог перколяции должен быть близок к классической величине $\varphi_{c}$ для сферических частиц, т. е. $\varphi_{c}=0.34$ [7]. Рассмотрим применимость указанных предельных случаев к нанокомпозитам ПУ/УНТ. Как известно [8], кольцеобразные формирования УНТ могут быть двух типов: „открытыми“ и „замкнутыми“. Первый тип структуры УНТ представляет собой геометрически дугу [9], второй является также геометрически аналогом окружности [8]. Граница между этими типами кольцеобразных структур $R_{\mathrm{CNT}}^{c r}$ определяется также из геометрических соображений согласно формуле [8]:

$$
R_{\mathrm{CNT}}^{c r}=\frac{L_{\mathrm{CNT}}}{2 \pi} .
$$

где $L_{\mathrm{CNT}}$ - длина углеродной нанотрубки.

В свою очередь, величину $R_{\mathrm{CNT}}$ можно определить с помощью следующего уравнения [10]:

$$
b_{\alpha}=5.6\left(R_{\mathrm{CNT}}^{2}-0.022\right) \text {. }
$$

где $R_{\mathrm{CNT}}$ задается в $\mathrm{mcm}$, а $b_{\alpha}$ является безразмерным параметром, характеризующим уровень межфазной ад- гезии в нанокомпозитах, который можно оценить с помощью следующего перколяционного соотношения [11]:

$$
\frac{E_{n}}{E_{m}}=1+11\left(c B_{\alpha} \varphi_{n}\right)^{17},
$$

где $c$ - константа, равная для УНТ 2.8 [11].

Оценки согласно уравнениям (4) и (5) дали значения $R_{\mathrm{CNT}}=0.67-0.29 \mathrm{mcm}$ в диапазоне $\varphi_{n}=0.024-0.301$ для рассматриваемых нанокомпозитов, тогда как при $L_{\mathrm{CNT}} \approx 10 \mathrm{mcm}$ величина $R_{\mathrm{CNT}}^{c r} \approx 1.5 \mathrm{mcm}$. Иначе говоря, в данном случае выполняется условие $R_{\mathrm{CNT}}<R_{\mathrm{CNT}}^{c r}$ и во всем интервале $\varphi_{n}$ кольцеобразные формирования УНТ в нанокомпозитах ПУ/УНТ являются ,замкнутыми“. Величину $\varphi_{n}$ для таких оценок можно о пределить согласно известной формуле [11]:

$$
\varphi_{n}=\frac{W_{n}}{\rho_{n}}
$$

где $W_{n}$ - массовое содержание нанонаполнителя, $\rho_{n}-$ его плотность, определяемая для углеродных нанотрубок следующим образом [11]:

$$
\rho_{n}=188\left(D_{\mathrm{CNT}}-d_{\mathrm{CNT}}\right)^{1 / 3}, \mathrm{~kg} / \mathrm{m}^{3},
$$

где $D_{\mathrm{CNT}}$ и $d_{\mathrm{CNT}}-$ наружный и внутренний диаметры углеродной нанотрубки, соответственно, задаваемые в нанометрах.

Далее можно определить расстояние между кольцеобразными формированиями $\lambda$ согласно следующему уравнению [11]:

$$
\lambda=\left[\left(\frac{4 \pi}{3 \varphi_{n}}\right)^{1 / 2}-2\right] R_{\mathrm{CNT}} .
$$

На рис. 1 приведена зависимость $\lambda\left(\varphi_{n}\right)$ для нанокомпозитов ПУ/УНТ, которая показала снижение $\lambda$ по мере роста $\varphi_{n}$ и при $\varphi_{n} \approx 0.34$ эта кривая асимптотически стремится к $\lambda=0$, что означает достижение порога перколяции $\varphi_{c}$. Это предполагает, что структура углеродных нанотрубок в полимерной матрице нанокомпозитов ПУ/УНТ может корректно моделироваться набором „замкнутых“ кольцеобразных формирований радиуса $R_{\mathrm{CNT}}$ при условии $\varphi_{n}<\varphi_{c}$ и поэтому для них корректно применение соотношения (2).

Далее необходимо определить показатель s в указанном соотношении. Как известно [12], величину $s$ можно определить из общего уравнения:

$$
\tilde{s}=\frac{s}{v}=\frac{D_{f}}{2}
$$

где $\tilde{s}-$ показатель, $v-$ стандартный индекс перколяции, равный 0.8 [7], $D_{f}-$ фрактальная размерность кольцеобразного формирования УНТ в полимерной матрице нанокомпозита. 


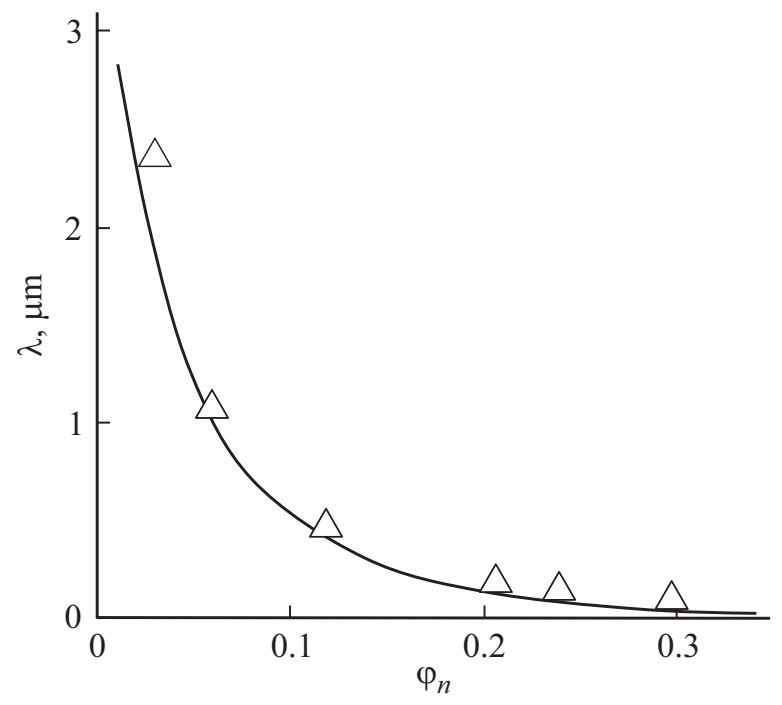

Рис. 1. Зависимость расстояния между кольцеобразными формированиями УНТ $\lambda$ от объемного содержания нанонаполнителя $\varphi_{n}$ для нанокомпозитов ПУ/УНТ.

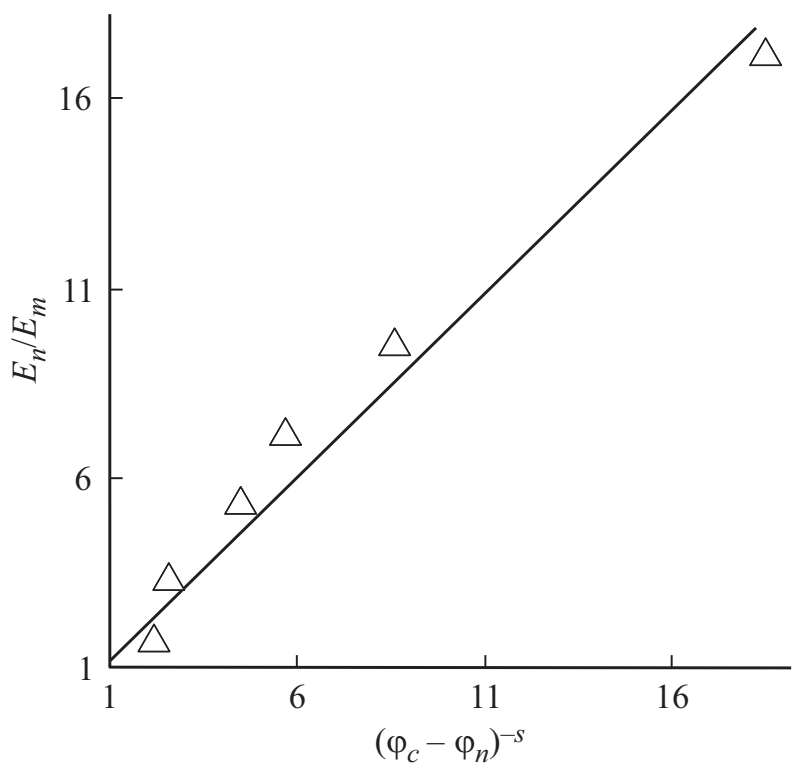

Рис. 2. Зависимость определенной экспериментально в настоящей статье по данным работы [3], рис. 4, $a$ степени усиления $E_{n} / E_{m}$ от параметра $\left(\varphi_{c}-\varphi_{n}\right)^{-s}$, полученного в рамках предела „термита“, для нанокомпозитов ПУ/УНТ.

Для указанных структур УНТ величину $D_{f}$ можно определить с помощью следующего уравнения [13]:

$$
R_{\mathrm{CNT}}=3.4\left(\varphi_{n}\right)^{-1 /\left(d-D_{f}\right)},
$$

где $R_{\mathrm{CNT}}$ дается в $\mathrm{nm}, d$ является евклидовой размерностью пространства, в котором рассматривается фрактал (очевидно, в нашем случае $d=3$ ).

Если пренебречь взаимопроникновением кластеров (кольцеобразных структур УНТ), основанием для чего служит график рис. 1 , то окончательно получим уравне- ние для определения показателя $s$ при любых $d$ [12]:

$$
s=\frac{\nu D_{f}}{2}=0.4 D_{f} .
$$

На рис. 2 приведено сравнение экспериментально полученных величин степени усиления $E n / E_{m}$ и теоретически определенного параметра $\left(\varphi_{c}-\varphi_{n}\right)^{-s}$ для нанокомпозитов ПУ/УНТ, соответствующее соотношению (2). Как следует из этого сравнения, между указанными параметрами наблюдается линейное соотношение и, более того, их равенство, что позволяет заменить в соотношении (2) знак пропорциональности на знак равенства. Иначе говоря, модель случайной сверхпроводящей сетки (ССС) или предел „термита“ корректно описывает степень усиления нанокомпозитов ПУ/УНТ.

И перколяционная модель [2], и модель „термита“ (CCC) имеют одну общую характерную точку соприкосновения - они демонстрируют зависимость свойств нанокомпозитов от структуры нанонаполнителя в полимерной матрице, независимо от типа нанонаполнителя. Кроме того, модель „термита“ (ССС) позволяет получить дополнительную информацию относительно факторов, влияющих на степень усиления нанокомпозитов. Так величину $s / v$ можно определить следующим образом [1]:

$$
\frac{s}{v}=d_{u}-(d-2),
$$

где $d_{u}-$ размерность неэкранированного (доступного для контакта с полимерной матрицей) периметра агрегата нанонаполнителя.

Следовательно, повышение размерности (доли) неэкранированного периметра агрегатов нанонаполнителя способствует повышению свойств нанокомпозитов. Кроме того, сравнение уравнений (11) и (12) демонстрирует прямую зависимость $d_{u}$ от $D_{f}$ и при $d=3$ получим

$$
d_{u}=\frac{D_{f}}{2}+1 .
$$

По поводу сделанных выше оценок следует сделать два замечания. Во-первых, плотность углеродных нанотрубок, определенная согласно уравнению (7), равна $825 \mathrm{~kg} / \mathrm{m}^{3}$, тогда как авторы [3] использовали для этой же цели произвольно выбранную величину $\rho_{n}=2000 \mathrm{~kg} / \mathrm{m}^{3}$. Использование этой величины $\rho_{n}$ для оценки объемного содержания УНТ $\rho_{n}$ согласно уравнению (6) и последующий расчет степени усиления $E_{n} / E_{m}$ согласно соотношению (2) приводит к пятикратному расхождению теории и эксперимента. В этой связи следует отметить, что в работе [14] для оценки величины $\varphi_{n}$ применялась гораздо более сложная формула по сравнению с уравнением (6), которая дала величину $\varphi_{n}$ существенно большую полученной авторами [3], но сравнимую с нашими оценками - максимальные значения $\varphi_{n}$ для нанокомпозитов ПУ/УНТ равны 0.340 и 0.301 соответственно.

Второе замечание касается получения высокомодульных нанокомпозитов полимер/углеродные нанотрубки. 
Авторы [15] получили с помощью специальных технологий нанокомпозиты бисмалеимид/углеродные нанотрубки, имеющие модуль упругости $E_{n}$ примерно $290 \mathrm{GPa}$, что равно соответствующему показателю для стали. Соотношение (2) позволяет оценить необходимые условия получения таких высокомодульных нанокомпозитов. Так, размерность нанонаполнителя (жгутов углеродных нанотрубок) должна приближаться к $D_{f} \approx 3$, содержание нанонаполнителя $\varphi_{n}-$ к порогу перколяции $\varphi_{c}$, а величина $E_{m}$ должна быть как можно выше. Отметим, что все эти критерии удовлетворяются для нанокомпозитов бисмалеимид/углеродные нанотрубки. Расчет по соотношению (2) или, точнее, уравнению (2), при следующих условиях: $E_{m}=4 \mathrm{GPa}, \varphi_{n}=0.31$ и $D_{f}=3$ дает величину $E_{n} \approx 269 \mathrm{GPa}$, что соответствует экспериментальным данным работы [15].

\section{3. Заключение}

Таким образом, полученные результаты продемонстрировали корректность моделирования нанокомпозитов полиуретан/углеродные нанотрубки как двухкомпонентных случайных смесей матричного полимера и нанонаполнителя. Количественное описание свойств этих нанокомпозитов дает модель случайной сверхпроводящей сетки или предел „термита“. Это описание является корректным, если локальная структура нанотрубок представлена их кольцеобразными формированиями. Структурным параметром, определяющим свойства нанокомпозитов, является фрактальная размерность кольцеобразных формирований углеродных нанотрубок. В рамках предложенной модели рассмотрены критерии получения высокомодульных нанокомпозитов полимер/углеродные нанотрубки.

\section{Конфликт интересов}

Авторы заявляют, что у них нет конфликта интересов.

\section{Список литературы}

[1] P. Meakin, T.A. Witten. Phys. Rev. A 28, 2985 (1983).

[2] Г.В. Козлов, И.В. Долбин. Прикл. физика 3, 96 (2017).

[3] H. Koerner, W. Liu, M. Alexander, P. Mirau, H. Dowty, R.A. Vaia. Polymer 46, 4405 (2005).

[4] A. Celzard, E. McRae, C. Deleuze, M. Dufort, G. Furdin, J.F. Mareche. Phys. Rev. B 53, 6209 (1996).

[5] M. Foygel, R.D. Morris, D. Anez, S. French, V.L. Sobolev. Phys. Rev. B 71, 104201 (2005).

[6] D.W. Schaefer, R.S. Justice. Macromolecules 40, 8501 (2007).

[7] Б.И. Шкловский, А.Л. Эфрос. УФН 117, 401 (1975).

[8] Г.В. Козлов, И.В. Долбин. Физика и химия стекла 45, 355 (2019).

[9] L.H. Shao, R.Y. Luo, S.L. Bai, J. Wang. Composite Struct. 87, 274 (2009).

[10] Г.В. Козлов, Ю.Г. Яновский, 3.М. Жирикова, В.З. Алоев, Ю.Н. Карнет. Механика композиционных материалов и конструкций 18, 131 (2012).
[11] А.К. Микитаев, Г.В. Козлов, Г.Е. Заиков. Полимерные нанокомпозиты: многообразие структурных форм и приложений. Наука, М. (2009). 278 c.

[12] A. Coniglio, H.E. Stanley. Phys. Rev. Lett. 52, 1068 (1984).

[13] А.К. Микитаев, Г.В. Козлов. ДАН 462, 41 (2015).

[14] D.W. Schaefer, J. Zhao, H. Dowty, M. Alexander, E.B. Orler. Soft Matter. 4, 2071 (2008).

[15] X. Wang, Z.Z. Yong, Q.W. Li, P.D. Bradford, W. Liu, D.S. Tucker, W. Cai, H. Wang, F.G. Yuan, Y.T. Zhu. Mater. Res. Lett. 1, 19 (2013).

Редактор Т.Н. Василевская 